\title{
pÿ2-Carotene degradation kinetics as affected by fat crystal network and solid/liquid ratio
}

\section{Calligaris, Sonia}

2018-03

pÿCalligaris , S , Valoppi , F , Barba , L , Anese , M \& Nicoli , M C 2018 , ' 2-Carotene degradation kinetics as affected by fat crystal network and solid/liquid ratio ', Food Research International , vol. 105 , pp. 599-604 . https://doi.org/10.1016/j.foodres.2017.11.062

http://hdl.handle.net/10138/308577

https://doi.org/10.1016/j.foodres.2017.11.062

cc_by_nc_nd

acceptedVersion

Downloaded from Helda, University of Helsinki institutional repository.

This is an electronic reprint of the original article.

This reprint may differ from the original in pagination and typographic detail.

Please cite the original version. 
Research International

Elsevier Editorial system(tm) for Food

Manuscript Draft

Manuscript Number: FOODRES-D-17-02299R1

Title: $\beta$-carotene degradation kinetics as affected by fat crystal network and solid/liquid ratio

Article Type: Research Paper

Keywords: $\beta$-Carotene; oxidation; fat crystal; kinetics; solid/liquid ratio.

Corresponding Author: Dr. Fabio Valoppi, Ph.D

Corresponding Author's Institution: Libera Università di Bolzano

First Author: Sonia Calligaris, Ph.D.

Order of Authors: Sonia Calligaris, Ph.D.; Fabio Valoppi, Ph.D; Luisa Barba, Dr. ; Monica Anese, Prof.; Maria Cristina Nicoli, Prof.

Abstract: The aim of this research was to study $\beta$-carotene degradation kinetics into lipid systems containing different fat crystal networks in the presence of increasing liquid oil amounts. To this purpose, fat blends containing liquid saturated medium chain triacylglycerols (MCT) with increasing content of saturated monoglycerides (MG), tripalmitin (PPP) and tristearin (SSS) were added with $0.6 \mathrm{mg} / \mathrm{g} \beta$-carotene. The fat crystal networks formed in the fat blends were characterized by using polarized light microscopy and synchrotron X-ray diffraction (XRD). In addition, $\beta$-carotene degradation was monitored during storage in the dark at $20^{\circ} \mathrm{C}$. Results highlighted that fat crystallization could differently affect $\beta$-carotene stability. In bulk SSS and PPP, $\beta$-carotene degradation proceeded at comparable rate, whereas when the saturated liquid oil MCT is included in the fat network, the rate of oxidation slightly decreased. Interestingly, the oxidation rate was not significantly affected by the solid/liquid ratio of the systems. A completely different behavior was observed in MG containing systems: the rate of $\beta$-carotene oxidation was in every case significantly lower than that observed in SSS and PPP containing samples. Also in this case, the MG protective effect was independent on its content in the fat mixtures. 
Dear Editor,

I would like to submit to your attention the manuscript entitled " $\beta$-carotene degradation kinetics as affected by fat crystal network and solid/liquid ratio" (Sonia Calligaris, Fabio Valoppi, Luisa Barba, Monica Anese, Maria Cristina Nicoli) for consideration for publication on Food Research International.

The aim of this research was to study $\beta$-carotene degradation kinetics into lipid systems containing different fat crystal networks in the presence of increasing liquid oil amounts. To this purpose, fat blends containing liquid saturated medium chain triacylglycerols (MCT) with increasing content of saturated monoglycerides (MG), tripalmitin (PPP) and tristearin (SSS) were added with $0.6 \mathrm{mg} / \mathrm{g} \beta$ carotene. The acquired results suggest that fat crystallization could differently affect $\beta$-carotene stability. In bulk SSS and PPP stored at $20{ }^{\circ} \mathrm{C} \beta$-carotene degradation proceeded at comparable rate, whereas when the liquid saturated oil MCT is included in the fat network, the rate of oxidation slightly decreased. Interestingly, the oxidation rate was not significantly affected by the solid/liquid ratio of the systems. Completely different behavior was observed in MG containing systems: the rate of $\beta$-carotene oxidation was in any case significantly lower than that observed in SSS and PPP containing samples. Also in this case, the MG protective effect was independent on its content in the fat mixtures.

This manuscript is the continuation of another article our group published on Food Research International in 2014 (Calligaris, S., Valoppi, F., Barba, L., Anese, M., \& Nicoli, M. C. (2014). Mutual effect of fat and beta-carotene on fat crystal network structure and carotenoid bleaching. Food Research International, 66, 257-263).

We would greatly appreciate your comments on the paper.

Best regards

Fabio Valoppi 


\section{Replies to referees' comments}

Ms. Ref. No.: FOODRES-D-17-02299

Title: <beta>-carotene degradation kinetics as affected by fat crystal network and solid/liquid ratio

(Please find grouped text pertaining to Reviewer 1 and 2; for each comment, reviewers' text is in italics, and answer/rebuttal text is normal)

Reviewers' comments:

Reviewer \#1: In my opinion the manuscript provides new interesting results for researcher in the field and it is well structured in all its sections, therefore I would recommend it for publication in the Food Research International journal.

We thank the reviewer for his/her appreciation of the manuscript.

Reviewer \#2: The objective of this research was to study the <beta>-carotene degradation kinetics into lipid systems containing different fat crystal networks in the presence of increasing liquid oil amounts.

The incorporation of <beta>-carotene into a fat crystal network and thus the choice of the lipid core to be a delivery system could deeply affect the chemical stability of the bioactive molecule. Knowledge about how the incorporation of bioactive compounds occurs in the lipid matrix and extremely important for the application in the development of foods, beverages, supplements, pharmaceuticals and cosmetics. In this way the manuscript after important contribution to study area of the food matrix.

We thank the reviewer for his/her appreciation of the manuscript. Below answers to reviewer comments.

Methods:

The method used for quantification of b-carotene is inappropriately described. It's not usual to use an external calibration curve for quantification of carotenoids in a spectrophotometer.

Why was cyclohexane used as a solvent? What is the molar extinction coefficient employed?

Even if $n$-hexane is mainly used in literature for $\beta$-carotene determination, also cyclohexane is used (Schierle et al., J AOAC Int., 2004; 87, 5, 1070-1082). $\beta$-carotene quantification was calculated using the extinction coefficient. The method was added in the relevant section (lines 182-191).

Results:

Figure 4 should show the B-carotene concentration on a log scale. At each point of degradation kinetics, the spot concentration should be normalized by the initial concentration of B-carotene. If the authors had used this scale they would realize that in both the SSS system and the PPP model, practically $100 \%$ of B-carotene was consumed in 4 days. Thus, the establishment of kinetics in this condition is incorrect. The experiment should have been repeated, with points withdrawn between 0 and 4 hours.

The figure was modified accordingly to reviewer suggestion. The experiments were repeated to increase the number of points in the first part of the curve. The first order rate constants were recalculated and reported in Table 2. Small differences in comparison to previous reported data were obtained. 
The authors are unable to discuss the cause of the loss of B-carotene, they only cite the action of oxygen reactive species, however, if only saturated fats were used as to explain the formation of these radicals in the absence of light?

Reading the literature, the autoxidation mechanism could be the main mechanism of $\beta$-carotene oxidation in the considered systems. Auto-oxidation could proceed at ambient temperature in the absence of light. The sentence was modified and references have been inserted (lines 288-290). 


\section{Highlights}

- Fat crystallization and structure differently affected $\beta$-carotene stability

- $\beta$-carotene degradation rate was higher in tripalmitin and tristearin containing samples compared to monoglyceride containing ones

- Solid/liquid ratio did not affect $\beta$-carotene degradation rates 


\section{$\beta$-carotene degradation kinetics as affected by fat crystal network and solid/liquid ratio}

Sonia Calligaris ${ }^{1}$, Fabio Valoppi ${ }^{1,2, *}$, Luisa Barba ${ }^{2}$, Monica Anese ${ }^{1}$, Maria Cristina Nicoli ${ }^{1}$

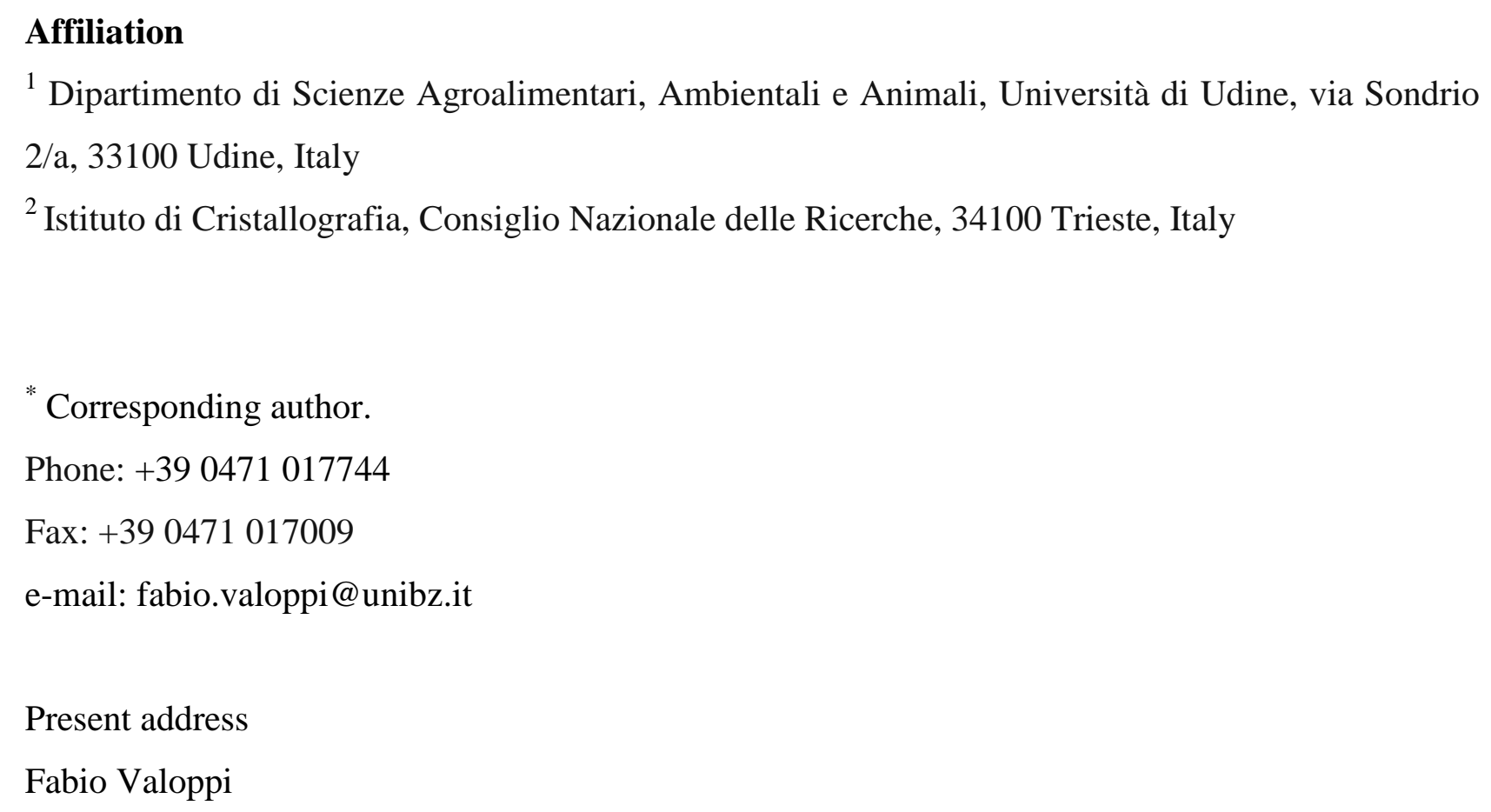
Italy 


\title{
Highlights
}

Fat crystallization and structure differently affected $\beta$-carotene stability

$\beta$-carotene degradation rate was higher in tripalmitin and tristearin containing samples compared to monoglyceride containing ones

Solid/liquid ratio did not affect $\beta$-carotene degradation rates

\begin{abstract}
The aim of this research was to study $\beta$-carotene degradation kinetics into lipid systems containing different fat crystal networks in the presence of increasing liquid oil amounts. To this purpose, fat blends containing liquid saturated medium chain triacylglycerols (MCT) with increasing content of saturated monoglycerides (MG), tripalmitin (PPP) and tristearin (SSS) were added with $0.6 \mathrm{mg} / \mathrm{g} \beta$ carotene. The fat crystal networks formed in the fat blends were characterized by using polarized light microscopy and synchrotron X-ray diffraction (XRD). In addition, $\beta$-carotene degradation was monitored during storage in the dark at $20{ }^{\circ} \mathrm{C}$. Results highlighted that fat crystallization could differently affect $\beta$-carotene stability. In bulk SSS and PPP, $\beta$-carotene degradation proceeded at comparable rate, whereas when the saturated liquid oil MCT is included in the fat network, the rate of oxidation slightly decreased. Interestingly, the oxidation rate was not significantly affected by the solid/liquid ratio of the systems. A completely different behavior was observed in MG containing systems: the rate of $\beta$-carotene oxidation was in every case significantly lower than that observed in SSS and PPP containing samples. Also in this case, the MG protective effect was independent on its content in the fat mixtures.
\end{abstract}

\section{Keywords}

$\beta$-Carotene, oxidation, fat crystal, kinetics, solid/liquid ratio.

\section{Introduction}

$\beta$-Carotene is one of the most studied compounds belonging to the carotenoids family. This is due not only to its provitamin A activity, being converted in the human intestine in retinol, but also to its well established antioxidant properties that have been associated to its health promoting capacity (Rao \& Rao, 2007; Ribeiro, Barreto, \& Coelho, 2011; Tapiero, Townsend, \& Tew, 2004). $\beta$ Carotene can be found in many fruits and vegetables derivatives or intentionally incorporated into foods to improve their health functionality as well as impart yellow/orange color. Lipid delivery systems, such as emulsions and microcapsules, have been indicated as suitable tools to incorporate lipophilic $\beta$-carotene into foods. These systems are made of a lipid phase, in which the carotenoid is 
solubilized, coated with surfactants or biopolymers (Chen, Fu, Hou, Guo, Wang, \& Yang, 2016; Lim, Griffin, \& Roos, 2014; Mahfoudhi \& Hamdi, 2015; Qian, Decker, Xiao, \& McClements, 2012; Xia, McClements, \& Xiao, 2015; Zhang, Xu, Jin, Shah, Li, \& Li, 2015). The lipid phase can vary in the triacylglycerol (TAG) composition showing different physical properties, from completely liquid oils to fats with different solid/liquid ratios as well as fat crystal network feature. The latter could contain TAG crystals organized into three main polymorphic forms: hexagonal $\alpha$, orthorhombic $\beta^{\prime}$, and triclinic $\beta$ (Hernqvist, 1988; Marangoni, Acevedo, Maleky, Co, Peyronel, Mazzanti, Quinn, \& Pink, 2012; Sato, 2001; Sato \& Ueno, 2011). These polymorphic forms are the subcell structures of the lamellar unit cell that is originated from the repetitive sequence of the acyl chains along the TAG long-chain axis (Sato \& Ueno, 2011). The lamellar structure builds up crystalline nanoplatelets that could aggregate into spherulites, which eventually originate a colloidal crystal network (Marangoni et al., 2012). TAGs spatial organizations have different thermodynamic stability and technological properties.

The incorporation of $\beta$-carotene into a fat crystal network and thus the choice of the lipid core to be included into a delivery system could deeply affect the chemical stability of the bioactive molecule. As well known, $\beta$-carotene is chemically unstable in foods and prone to isomerization and oxidation, leading to color fading as well as loss of healthy properties (Namitha \& Negi, 2010). Some authors evidenced that the degradation rate of lipophilic bioactive molecules could be retarded in solid lipid nanoparticles compared to emulsions with liquid core (Nik, Langmaid, \& Wright, 2012; Relkin, Jung, \& Ollivon, 2009; Zhang, Hayes, Chen, \& Zhong, 2013). However, opposite results have been also reported (Cornacchia \& Roos, 2011; Helgason, Awad, Kristbergsson, Decker, McClements, \& Weiss, 2009; Qian, Decker, Xiao, \& McClements, 2013). Regarding $\beta$-carotene, in a previous research we demonstrated that $\beta$-carotene stability is significantly affected by the fat crystal network architecture (Calligaris, Valoppi, Barba, Anese, \& Nicoli, 2014). $\beta$-carotene resulted more stable in bulk monoglyceride than in presence of tripalmitin (PPP) and tristearin (SSS) crystals, highlighting the critical role of the type of crystallizing molecule (Calligaris et al., 2014). This result was attributed to the different location and compartmentalization of the carotenoid in the fat crystal network.

The aim of this research was to study the $\beta$-carotene degradation kinetics into lipid systems containing different fat crystal networks in the presence of increasing liquid oil amounts. To this purpose, fat blends containing liquid saturated medium chain triacylglycerols (MCT) with increasing content of saturated monoglycerides (MG), tripalmitin (PPP) and tristearin (SSS) were added with $0.6 \mathrm{mg} / \mathrm{g} \beta$-carotene. All the selected lipid matrices were composed of saturated fatty acids to avoid the possible concurrent oxidation of the lipid matrices besides that of $\beta$-carotene. The 
fat crystal network formed in the fat blends were characterized by using polarized light microscopy and synchrotron X-ray diffraction (XRD). In addition, $\beta$-carotene degradation was monitored during storage in the dark at $20^{\circ} \mathrm{C}$.

\section{Materials and methods}

\subsection{Materials}

Myverol $^{\mathrm{TM}}$ saturated monoglyceride (MG) (fatty acid composition: 1.4\% C14:0, 59.8\% C16:0, $38.8 \% \mathrm{C} 18: 0$; melting point $68.05 \pm 0.5^{\circ} \mathrm{C}$ ) was kindly donated by Kerry Ingredients and Flavour (Bristol, United Kingdom). Tripalmitin (PPP; purity $\geq 85 \%$ ), tristearin (SSS; purity $\geq 85 \%$ ), and crystalline $\beta$-carotene (purity $\geq 93 \%$ ) were purchased from Sigma Aldrich (Milan, Italy). Miglyol $812 \mathrm{~N}$ (MCT; fatty acid composition: 57.0\% C8:0, 43.0\% C10:0) was kindly provided by Cremer Oleo GmbH \& Co. KG (Hamburg, Germany).

\subsection{Sample preparation}

Around $2 \mathrm{~g}$ of mixtures containing solid fats (MG, PPP, or SSS) and liquid triacylglycerols (MCT) at different solid fat content $(100,90,70,50,40,30,20,10,15,5$ and $0 \%$ w/w) added or not with $0.6 \mathrm{mg} / \mathrm{g}$ of crystalline $\beta$-carotene were weighted into $20-\mathrm{mL}$ vials, saturated with nitrogen and hermetically sealed. Samples were heated at $80^{\circ} \mathrm{C}$ in the dark until completely transparent solutions were obtained (i.e. visibly free of dispersed materials). Finally, samples were cooled from 80 to 20 ${ }^{\circ} \mathrm{C}$ at $2{ }^{\circ} \mathrm{C} / \mathrm{min}$ using a PC200-G50 temperature controlled water bath (Thermo Fisher Scientific, Waltham, USA) and ice. Samples were stored at $20{ }^{\circ} \mathrm{C}$ for 24 hours before analysis.

Further samples were prepared for $\beta$-carotene degradation kinetic study. Around $20 \mathrm{~g}$ of mixtures containing solid fats (MG, PPP, or SSS) and liquid triacylglycerols (MCT) at different solid fat content $(100,70,50,30,10$, and $0 \% \mathrm{w} / \mathrm{w})$ added with $0.6 \mathrm{mg} / \mathrm{g}$ of crystalline $\beta$-carotene were weighted in $100 \mathrm{~mL}$ amber bottles, saturated with nitrogen and sealed. Samples were heated at 80 ${ }^{\circ} \mathrm{C}$ and stirred with a magnetic rod in the dark until completely transparent solutions were obtained (i.e. visibly free of dispersed materials). Approximately $5.5 \mathrm{~g}$ of molten sample was placed into 6$\mathrm{cm}$ diameter Petri dishes and covered with plastic caps. Petri dishes were then cooled at room temperature and stored for up to 14 days in a temperature controlled incubator in the dark at $20{ }^{\circ} \mathrm{C}$.

\subsection{Polarized light microscopy}

One drop of the sample was placed in the middle of a glass slide and a glass cover slip was centered above the drop. The glass slide was transferred to the microscope allocation previously heated at 80 ${ }^{\circ} \mathrm{C}$ and fully melted for 10 min using a Linkam CSS450 temperature controller (Linkam Scientific 
Instruments, Surrey, UK). Sample was then cooled to $20^{\circ} \mathrm{C}$ at $2{ }^{\circ} \mathrm{C} / \mathrm{min}$ before being imaged at 200× with a polarized light (PL) optical microscope (Leica DM 2000, Leica Microsystems, Heerburg, Switzerland) connected with a Leica EC3 digital camera (Leica Microsystems, Heerburg, Switzerland). Images were acquired and processed using the application software Leica Suite LAS EZ (Leica Microsystems) and saved in jpeg format resulting in $2048 \times 1536$ pixels corresponding to $597 \times 448 \mu \mathrm{m}$.

\subsection{Crystal size}

Crystal size was computed on selected images using Image-Pro® Plus (ver. 6.3, Media Cybernetics, Inc., Bethesda, MD, USA). A spatial calibration was firstly performed using the $100 \mu \mathrm{m}$ reference line obtained with the microscope application software. Then, a line was manually drew over each crystal and the length was automatically calculated.

\subsection{Fractal dimension}

Polarized light micrographs were used to compute the fractal dimension of the crystals. In particular, images were converted into gray scale $(8$ bit) and thresholded using Image-Pro® Plus (ver. 6.3, Media Cybernetics, Inc., Bethesda, USA) in order to allow all the crystalline material present in the image to be highlighted. The box-counting fractal dimension $\left(\mathrm{D}_{\mathrm{b}}\right)$ was calculated using ImageJ ver. 1.51a (National Institutes of Health, USA) with the plugin FracLac (ver. 2015sep090313a9330, Charles Sturt University, Bathurst, Australia). The setting parameters used were the number of positions for grids $G$, equal to 30 , locked black background, and the maximum size of the crystal, set at $45 \%$ of the total image area.

\subsection{Synchrotron X-ray diffraction (XRD)}

$\mathrm{X}$-ray diffraction patterns were recorded at the X-ray diffraction beam-line 5.2 at the Synchrotron Radiation Facility Elettra in Trieste (Italy). The X-ray beam emitted by the wiggler source on the Elettra $2 \mathrm{GeV}$ electron storage ring was monochromatized by a $\mathrm{Si}(111)$ double crystal monochromator, focused on the sample and collimated by a double set of slits giving a spot size of $0.2 \times 0.2 \mathrm{~mm}$. An aliquot of sample was lodged into a nylon pre-mounted cryoloop $20 \mathrm{~mm}$ for crystallographic experiments (0.7-1.0 mm) (Hampton Research HR4-965, Aliso Veijo, CA, USA). Sample temperature was controlled by means of a 700 series cryocooler (Oxford Cryosystems, Oxford, UK) with an accuracy of $\sim{ }^{\circ} \mathrm{C}$. Analyses were performed at $20^{\circ} \mathrm{C}$. Data were collected at a photon energy of $8.856 \mathrm{keV}(\lambda=1.4 \AA)$, using a $2 \mathrm{M}$ Pilatus silicon pixel X-ray detector (DECTRIS Ltd., Baden, Switzerland). Bidimensional patterns collected with Pilatus were calibrated 
by means of $\mathrm{a} \mathrm{LaB}_{6}$ standard and integrated using the software FIT2D (Hammersley, Svensson, Hanfland, Fitch, \& Hausermann, 1996). The peak position and full width at half of the maximum peak height $(F W H M)$ were evaluated by means of the program Winplotr (Roisnel \& RodríguezCarvajal, 2000); indexing of the XRD patterns obtained by the crystalline phases was performed using Checkcell (Laugier \& Bochu, 2000).

\subsection{Crystalline domain size calculations}

From the small-angle X-ray diffraction region (SAXD), the crystalline domain size $(\xi)$ was calculated using two approaches: (i) the analysis introduced by Warren and Averbach (1950) and Warren and Averbach (1952), and (ii) the Scherrer equation (Acevedo \& Marangoni, 2010). The first approach is based on the separation of the contributions from finite crystallite dimensions and disorder, considering the long-range ordering capability showed by these compounds (testified by the many orders detectable of the $00 l$ crystallographic direction). The average volume weighted crystallite dimension was calculated in the assumption of $25 \%$ peak shape Gaussian component using the methodology proposed by Enzo, Fagherazzi, Benedetti, and Polizzi (1988). In this case, the instrumental function was considered negligible with respect to the sample contribution to peak broadening considering the collimation and dimension properties of synchrotron X-ray beam. The second approach is based on the relationship between the $F W H M$ in radians and the diffraction angle $(\theta)$ as shown in eq. 1 .

$\xi=\frac{K \cdot \lambda}{F W H M \cdot \cos \theta}$

where $K$ is the shape factor which usually is set at 0.9 for crystallites with unknown shape, and $\lambda$ is the wave length of the $\mathrm{X}$-ray.

\section{$2.8 \beta$-carotene concentration}

Samples containing $\beta$-carotene were grinded using a mortar and a pestle. Subsequently, $\beta$-carotene was extracted using cyclohexane as solvent in an ultrasonic bath. The residual concentration of $\beta$ carotene at time $t\left(C_{t}\right)$ was measured spectrophotometrically at $450 \mathrm{~nm}$ by means of a UV/VIS spectrophotometer Shimadzu UV-250 1 PC (Shimadzu, Kyoto, Japan) and calculated accordingly to the formula:

$C_{t}=\frac{A}{E_{1 \mathrm{~cm}}^{1 \%} \cdot m \cdot d}$ 
where $A$ equals the measured absorbance at $450 \mathrm{~nm}, E_{1 \mathrm{~cm}}^{1 \%}$ the extinction coefficient of $\beta$-carotene in cyclohexane $\left(2500 \mathrm{~g}^{-1} \mathrm{Lcm}^{-1}\right), m$ the mass $(\mathrm{g})$ of the sample, and $d$ the length $(\mathrm{cm})$ of the cuvette. Finally, $\beta$-carotene residual concentration was normalized against its initial concentration $\left(C_{t} / C_{0}\right)$.

\subsection{Data analysis}

All determinations were expressed as the mean \pm standard error (SE) of at least two measurements from two experiment replicates $(\mathrm{n} \geq 4$ ). Statistical analysis was performed using R v. 3.0.2 (The R foundation for Statistical Computing). Bartlett's test was used to check the homogeneity of variance, one-way ANOVA was carried out and Tukey-test was used as posthoc test to determine statistical significant differences among means $(p<0.05)$. Linear regression analysis by least squares minimization was performed using GraphPad Prism ver. 5.03 (GraphPad Software, San Diego, USA). The goodness of fit was evaluated on the basis of statistical parameters of fitting $\left(\mathrm{R}_{\text {adj }}^{2}, p\right.$, standard error) and the residual analysis.

\section{Result and discussion}

\subsection{Structural properties of saturated lipid mixtures}

PL micrographs of lipid blends containing increasing concentrations of MG, PPP, and SSS in liquid MCT are reported in Figure 1. The dark zones in the images refer to the isotropic liquid MCT, whereas the bright areas are anisotropic crystals of MG, PPP, or SSS. As expected, the dimensions of the crystalline structures increased with increasing the solid fat content and in general were bigger in MG containing samples. At $10 \%$ fat concentration, all samples showed structures with dimensions of about 20-40 $\mu \mathrm{m}$. The aggregate size increased up to 70-120 $\mu \mathrm{m}$ at higher concentrations. Up to a critical concentration value, a crowded network with big crystalline aggregates was obtained and a uniform crystalline mass can be observed. This critical value was $70 \%$ and 50\% for PPP and SSS containing samples, respectively; whereas MG containing samples did not show a defined critical concentration value. Moreover, different crystal morphologies can be observed depending on solid lipid type and concentration: platelet-like structures were noted in MG containing samples; spherulites composed of small crystals in 10 and 30\% SSS containing samples, and platelet-like crystals aggregated into spherulites in 10\%, 30\% and 50\% PPP containing samples. To better investigate the structural organization of fats in MCT, fractal dimension was computed from PL micrographs using the box-counting method (Ahmadi, Wright, \& Marangoni, 2008) (Figure 2). As expected, crystal aggregates size increased with the increasing of the solid fat content. A limit vale of about 1.84-1.86 was reached at different solid fat content depending on fat type. Both SSS and PPP containing mixtures reached the plateau value at concentrations higher than 
$50 \%$ and $70 \%$, respectively, corresponding to the formation of the uniform crystalline mass (Figure 1). It is noteworthy that the kinetics of fractal dimension changes were different for the three fat systems. PPP containing samples slowly and progressively reached the plateau value probably due to a lower ability of platelet-like crystals to form the crystal network in comparison to SSS spherulites. By contrast, MG containing mixtures rapidly approached to the plateau value, indicating a higher network forming ability than SSS and PPP molecules. $\mathrm{XRD}$ analysis was performed to determine the polymorphic form, the interplanar distance between the reflecting planes, and the crystalline domain size $(\xi)$ of MG-, SSS- and PPP-MCT mixtures. As $\frac{14}{15} 32$ an example, Figure 3 shows the integrated patterns with relevant peak position of the crystallographic reflecting planes belonging to the $00 l$ family, along with their relative sections of the bidimensional patterns, of 100\% MG (A), 50\% SSS (B), 10\% PPP (C). The polymorphic forms, the interplanar distance $\left(d_{001}\right)$ as well as the calculated crystal size domain are reported in Table 1 . Hexagonal $\alpha$ form was observed in the $100 \%$ MG, PPP, and SSS containing samples, while the $70 \%, 50 \%, 30 \%$ and 10\% MG and PPP containing samples as well as the 10\% SSS containing one showed $\beta$ crystals. By contrast, 30\%, 50\% and 70\% concentrations of SSS containing samples showed $\beta^{\prime}$ form polymorph. In general terms, the less thermodynamically stable polymorphs were detected in bulk neat fats. Decreasing the fat and increasing the liquid oil amounts more stable polymorphic crystals formed, in agreement with the literature (Ahmadi et al., 2008; Wright, Batte, $\&$ Marangoni, 2005). This can be attributed to a slower nucleation from hot melt that allowed MG, PPP, and SSS molecules to organize in the best thermodynamic polymorph depending on solid fat concentration. The interplanar distances $\left(d_{001}\right)$ reported in Table 1 were in good agreement with data reported in the literature (Calligaris et al., 2014; Valoppi, Calligaris, Barba, Šegatin, Poklar Ulrih, $\&$ Nicoli, 2017). As expected, $\alpha$ form showed a higher $d_{001}$ than that of other polymorphic forms, irrespectively of solid fat content. According to Hernqvist (1988), the moieties of triglycerides or monoglycerides are perpendicular to the crystallographic reflecting plane in the $\alpha$ form. Thus, the tilting angles of the $\beta$ and $\beta^{\prime}$ forms can be geometrically calculated. It was found that MG samples showed a tilting angle of the carbon chain respect to the reflecting planes of $71.3-68.3^{\circ}$, whereas PPP and SSS showed tilting angles between $64.0-62.7^{\circ}$ and $65.2-64.2^{\circ}$, respectively.

Regarding the $\xi$ computation, WA method and Scherrer formula were used (Acevedo \& Marangoni, 2010; Warren \& Averbach, 1950, 1952). It is known in the literature that Scherrer formula derivation is based on the assumption that the crystalline shape is cubic and the diffracted beam shape is considered Gaussian. This assumption simplifies the derivation process but introduces an important drawback that is the difficulty in relating the coordination length to the "true" dimensions of the crystallites (Scardi, Leoni, \& Delhez, 2004; Warren, 1990). On the other hand, the WA 
approach does not take into account strain anisotropy effects and systematic errors in evaluating the peak position and FWHM (Scardi et al., 2004). Although aware of these limitations, the $\xi$ was computed. A discrepancy in the crystalline domain size between WA and Scherrer approaches was actually noted. In particular, smaller values were obtained by applying the WA method than those computed by using the Scherrer formula. Besides these discrepancies, it was found that crystallite dimensions were dependent on the polymorphic form (and thus the lamellar thickness) rather than on the solid fat content. In general, the following trend in domain size was found: PPP > MG > SSS.

Crystalline $\beta$-carotene was then mixed with melted systems at a concentration of $0.6 \mathrm{mg} / \mathrm{g}$. At this concentration, the bioactive molecule was totally solubilized in the lipid matrix since its solubility in oil was about 0.12\% (Borel, Grolier, Armand, Partier, Lafont, Lairon, \& Azais-Braesco, 1996). No changes in crystal size, fractal dimension, polymorphic form, interplanar distance between the reflecting planes and crystalline domain size were found (data not shown). These results are apparently in contrast with previous data showing that the presence of $5 \%$ of $\beta$-carotene in neat MG, PPP, and SSS was able to modify the fat crystallization behavior (Calligaris et al., 2014). It can be inferred that the small amount of the bioactive molecule used in this study was not sufficient to affect the structural properties of the fat blends.

\section{2 $\beta$-carotene stability in lipid blends}

Figure 4 shows the changes of $\beta$-carotene concentration in $100 \%$ containing SSS, PPP, MCT and $\mathrm{MG}$ as a function of storage time at $20^{\circ} \mathrm{C}$. A sharp and rapid decrease of $\beta$-carotene content was observed in SSS and PPP samples, which was completely degraded after 4 days of storage. The carotenoid degradation resulted slower in MCT and its concentration was no more detectable after about 14 days. Finally, $\beta$-carotene appeared particularly stable in MG, as well evidenced by the slight concentration decrease during storage, in agreement with previous data (Calligaris et al., 2014).

$\beta$-Carotene degradation can be ascribed to a pseudo first order kinetics, in agreement with the literature (Dutta, Dutta, Raychaudhuri, \& Chakraborty, 2006; Stefanovich \& Karel, 1982). To highlight the effect of the solid/liquid ratio in fat blends, similar experiments were performed on samples containing increasing contents of crystalline MG, PPP, and SSS in liquid MCT. Table 2 shows the estimated pseudo first order rate constants of $\beta$-carotene degradation. As already mentioned, the selected lipid matrices contained saturated fatty acid moiety in the molecules of triacylglycerols or monoglycerides to reduce as much as possible the presence of peroxide radicals deriving from the oxidation of fatty acids. Thus, the main expected mechanism of $\beta$-carotene 
degradation was the reaction between $\beta$-carotene and dissolved oxygen through autoxidation mechanism (Britton, 1995; Burton, Daroszewski, Nickerson, Johnston, Mogg, \& Nikiforov, 2014). In liquid MCT ( $0 \%$ solid fat), the rate constant of $\beta$-carotene degradation was $-0.284 \pm 0.062$ days $^{-1}$. The presence of SSS and PPP crystals in MCT did not significantly modify the kinetics of $\beta$ carotene degradation at any tested concentration up to $70 \%(\mathrm{w} / \mathrm{w})$. It can be hypothesized that, during fat crystallization, $\beta$-carotene remained prevalently dissolved in the liquid MCT not participating to the fat crystal formation. In these conditions, oxygen can freely dissolve and diffuse in the liquid matrix event at the highest solid fat content. The carotenoid oxidation rate increased in the neat crystallized PPP and SSS, indicating that the bioactive molecule was more easily attainable by oxygen. As previously reported by our group (Calligaris et al., 2014), in these systems $\beta$ carotene could be included in the crystal network of PPP and SSS. No significant differences in reaction rates were found between PPP and SSS containing samples although the crystal morphology was different.

Completely different $\beta$-carotene degradation rates were observed in MG containing samples. It is well evident from the results reported in Table 2 that the presence of MG hindered the $\beta$-carotene degradation at all tested concentrations. Interestingly, the MG protective effect was independent on its content in the fat mixtures: the crystalline network established by MG at $10 \%$ concentration was enough to significantly slow down $\beta$-carotene degradation reaction. It was previously observed that $\beta$-carotene at $5 \%$ concentration formed amorphous clusters in crystallized MG (Calligaris et al., 2014). It can be hypothesized that $\beta$-carotene would be located within MG crystals even at the low concentration considered in this study, thus allowing its protection.

\section{Conclusions}

The acquired results suggest that fat crystallization could differently affect $\beta$-carotene stability. In bulk SSS and PPP stored at $20{ }^{\circ} \mathrm{C} \beta$-carotene degradation proceeded at comparable rate, whereas when the liquid saturated oil MCT is included in the fat network, the rate of oxidation slightly decreased. Interestingly, the oxidation rate was not significantly affected by the solid/liquid ratio of the systems. Completely different behavior was observed in MG containing systems: the rate of $\beta$ carotene oxidation was in any case significantly lower than that observed in SSS and PPP containing samples. Also in this case, the MG protective effect was independent on its content in the fat mixtures. These results clearly seem to be crucial in the attempt to design efficient delivery systems highlighting the importance of the choice of the lipid carrier in the attempt to protect $\beta$ carotene against oxidation. 


\section{Acknowledgments}

This research was partially supported by the project "S.H.A.R.M. - Supporting Human Assets in Research and Mobility" (project number: FP1373849009) financed by Friuli Venezia Giulia Region and European social fund.

\section{References}

Acevedo, N. C., \& Marangoni, A. G. (2010). Characterization of the Nanoscale in Triacylglycerol Crystal Networks. Crystal Growth \& Design, 10, 3327-3333.

Ahmadi, L., Wright, A. J., \& Marangoni, A. G. (2008). Chemical and enzymatic interesterification of tristearin/triolein-rich blends: Microstructure and polymorphism. European Journal of Lipid Science and Technology, 110, 1025-1034.

Borel, P., Grolier, P., Armand, M., Partier, A., Lafont, H., Lairon, D., \& Azais-Braesco, V. (1996). Carotenoids in biological emulsions: solubility, surface-to-core distribution, and release from lipid droplets. Journal of Lipid Research, 37, 250-261.

Britton, G. (1995). Structure and properties of carotenoids in relation to function. The FASEB Journal, 9, 1551-1558.

Burton, G. W., Daroszewski, J., Nickerson, J. G., Johnston, J. B., Mogg, T. J., \& Nikiforov, G. B. (2014). $\beta$-Carotene autoxidation: oxygen copolymerization, non-vitamin A products, and immunological activity. Canadian Journal of Chemistry, 92, 305-316.

Calligaris, S., Valoppi, F., Barba, L., Anese, M., \& Nicoli, M. C. (2014). Mutual effect of fat and beta-carotene on fat crystal network structure and carotenoid bleaching. Food Research International, 66, 257-263.

Chen, X. W., Fu, S. Y., Hou, J. J., Guo, J., Wang, J. M., \& Yang, X. Q. (2016). Zein based oil-inglycerol emulgels enriched with beta-carotene as margarine alternatives. Food Chemistry, $211,836-844$.

Cornacchia, L., \& Roos, Y. H. (2011). Stability of beta-carotene in protein-stabilized oil-in-water delivery systems. Journal of Agricultural and Food Chemistry, 59, 7013-7020.

Dutta, D., Dutta, A., Raychaudhuri, U., \& Chakraborty, R. (2006). Rheological characteristics and thermal degradation kinetics of beta-carotene in pumpkin puree. Journal of Food Engineering, 76, 538-546.

Enzo, S., Fagherazzi, G., Benedetti, A., \& Polizzi, S. (1988). A profile-fitting procedure for analysis of broadened X-ray diffraction peaks. I. Methodology. Journal of Applied Crystallography, $21,536-542$. 
Hammersley, A. P., Svensson, S. O., Hanfland, M., Fitch, A. N., \& Hausermann, D. (1996). Twodimensional detector software: From real detector to idealised image or two-theta scan. High Pressure Research, 14, 235-248.

Helgason, T., Awad, T. S., Kristbergsson, K., Decker, E. A., McClements, D. J., \& Weiss, J. (2009). Impact of surfactant properties on oxidative stability of beta-carotene encapsulated within solid lipid nanoparticles. Journal of Agricultural and Food Chemistry, 57, 80338040.

Hernqvist, L. (1988). Crystal structures of fats and fatty acids. In N. Garti \& K. Sato (Eds.), Crystallization and polymorphism of fats and fatty acids (pp. 97-137). New York: Marcel Dekker, Inc.

Laugier, J., \& Bochu, B. (2000). CHECKCELL. http://www.CCP14.ac.uk/tutorial/lmgp/. Retrieved 10 May 2016, from http://www.CCP14.ac.uk/tutorial/lmgp/

Lim, A. S. L., Griffin, C., \& Roos, Y. H. (2014). Stability and loss kinetics of lutein and $\beta$-carotene encapsulated in freeze-dried emulsions with layered interface and trehalose as glass former. Food Research International, 62, 403-409.

Mahfoudhi, N., \& Hamdi, S. (2015). Kinetic Degradation and Storage Stability of $\beta$-Carotene Encapsulated by Freeze-Drying Using Almond Gum and Gum Arabic as Wall Materials. Journal of Food Processing and Preservation, 39, 896-906.

Marangoni, A. G., Acevedo, N., Maleky, F., Co, E., Peyronel, F., Mazzanti, G., Quinn, B., \& Pink, D. (2012). Structure and functionality of edible fats. Soft Matter, 8, 1275-1300.

Namitha, K. K., \& Negi, P. S. (2010). Chemistry and biotechnology of carotenoids. Critical Reviews in Food Science and Nutrition, 50, 728-760.

Nik, A. M., Langmaid, S., \& Wright, A. J. (2012). Nonionic surfactant and interfacial structure impact crystallinity and stability of beta-carotene loaded lipid nanodispersions. Journal of Agricultural and Food Chemistry, 60, 4126-4135.

Qian, C., Decker, E. A., Xiao, H., \& McClements, D. J. (2012). Nanoemulsion delivery systems: influence of carrier oil on beta-carotene bioaccessibility. Food Chemistry, 135, 1440-1447.

Qian, C., Decker, E. A., Xiao, H., \& McClements, D. J. (2013). Impact of lipid nanoparticle physical state on particle aggregation and beta-carotene degradation: Potential limitations of solid lipid nanoparticles. Food Research International, 52, 342-349.

Rao, A. V., \& Rao, L. G. (2007). Carotenoids and human health. Pharmacological Research, 55, 207-216.

Relkin, P., Jung, J. M., \& Ollivon, M. (2009). Factors affecting vitamin degradation in oil-in-water nano-emulsions. Journal of Thermal Analysis and Calorimetry, 98, 13-18. 
Ribeiro, B. D., Barreto, D. W., \& Coelho, M. A. Z. (2011). Technological Aspects of $\beta$-Carotene Production. Food and Bioprocess Technology, 4, 693-701.

Roisnel, T., \& Rodríguez-Carvajal, J. (2000). WinPLOTR: a Windows tool for powder diffraction patterns analysis. Materials Science Forum, Proceedings of the Seventh European Powder Diffraction Conference (EPDIC 7), Eds. R. Delhez \& E. J. Mittenmeijer, pp. 118-123.

Sato, K. (2001). Crystallization behaviour of fats and lipids - a review. Chemical Engineering Science, 56, 2255-2265.

Sato, K., \& Ueno, S. (2011). Crystallization, transformation and microstructures of polymorphic fats in colloidal dispersion states. Current Opinion in Colloid \& Interface Science, 16, 384390.

Scardi, P., Leoni, M., \& Delhez, R. (2004). Line broadening analysis using integral breadth methods: a critical review. Journal of Applied Crystallography, 37, 381-390.

Stefanovich, A. F., \& Karel, M. (1982). Kinetics of beta-carotene degradation at temperatures typical of air drying of foods. Journal of Food Processing and Preservation, 6, 227-242.

Tapiero, H., Townsend, D. M., \& Tew, K. D. (2004). The role of carotenoids in the prevention of human pathologies. Biomedicine \& Pharmacotherapy, 58, 100-110.

Valoppi, F., Calligaris, S., Barba, L., Šegatin, N., Poklar Ulrih, N., \& Nicoli, M. C. (2017). Influence of oil type on formation, structure, thermal, and physical properties of monoglyceride-based organogel. European Journal of Lipid Science and Technology, 119, 1500549.

Warren, B. E. (1990). X-Ray Diffraction (B. E. Warren Ed.). New York: Dover Pubblications, Inc.

Warren, B. E., \& Averbach, B. L. (1950). The Effect of Cold- Work Distortion on X- Ray Patterns. Journal of Applied Physics, 21, 595-599.

Warren, B. E., \& Averbach, B. L. (1952). The Separation of Cold- Work Distortion and Particle Size Broadening in X- Ray Patterns. Journal of Applied Physics, 23, 497-497.

Wright, A. J., Batte, H. D., \& Marangoni, A. G. (2005). Effects of canola oil dilution on anhydrous milk fat crystallization and fractionation behavior. Journal of Dairy Science, 88, 1955-1965.

Xia, Z., McClements, D. J., \& Xiao, H. (2015). Influence of physical state of beta-carotene (crystallized versus solubilized) on bioaccessibility. Journal of Agricultural and Food Chemistry, 63, 990-997.

Zhang, C., Xu, W., Jin, W., Shah, B. R., Li, Y., \& Li, B. (2015). Influence of anionic alginate and cationic chitosan on physicochemical stability and carotenoids bioaccessibility of soy protein isolate-stabilized emulsions. Food Research International, 77, 419-425. 
425 Zhang, L., Hayes, D. G., Chen, G., \& Zhong, Q. (2013). Transparent dispersions of milk-fat-based 426 nanostructured lipid carriers for delivery of beta-carotene. Journal of Agricultural and Food Chemistry, 61, 9435-9443. 
429 Table 1. Polymorphic form, interplanar distance between reflecting planes $\left(d_{001}\right)$, crystalline $4 \sqrt{30}$ domain size $(\xi)$ calculated using WA method and Scherrer formula for samples containing different 4,31 concentrations of MG, PPP, and SSS in liquid MCT.

\begin{tabular}{lccccc}
\hline Solid Fat & Percentage & Polymorph & $d_{001}$ & \multicolumn{2}{c}{$\xi(\AA)$} \\
\cline { 5 - 6 }$(\%$ w/w $)$ & & $(\AA)$ & WA & Scherrer \\
\hline MG & 10 & $\beta$ & 46.25 & 196.9 & 386.9 \\
MG & 30 & $\beta$ & 45.83 & 193.4 & 391.0 \\
MG & 50 & $\beta$ & 46.73 & 222.3 & 450.0 \\
MG & 70 & $\beta$ & 46.23 & 205.2 & 413.1 \\
MG & 100 & $\alpha$ & 49.32 & 274.3 & 474.8 \\
PPP & 10 & $\beta$ & 39.19 & 249.1 & 634.9 \\
PPP & 30 & $\beta$ & 39.21 & 264.4 & 652.9 \\
PPP & 50 & $\beta$ & 39.62 & 222.3 & 578.3 \\
PPP & 70 & $\beta$ & 39.66 & 228.2 & 604.4 \\
PPP & 100 & $\alpha$ & 44.12 & 201.0 & 458.0 \\
SSS & 10 & $\beta$ & 43.43 & 174.3 & 376.6 \\
SSS & 30 & $\beta^{\prime}$ & 43.76 & 189.4 & 421.3 \\
SSS & 50 & $\beta^{\prime}$ & 43.78 & 191.2 & 424.2 \\
SSS & 70 & $\beta^{\prime}$ & 43.68 & 185.8 & 412.2 \\
SSS & 100 & $\alpha$ & 48.24 & 195.2 & 379.2 \\
\hline
\end{tabular}

(3) 
433 $\frac{1}{434}$

Table 2. Pseudo first order reaction rate of $\beta$-carotene degradation in fat blend containing increasing content of solid MG, PPP, and SSS in MCT.

\begin{tabular}{|c|c|c|c|c|c|c|}
\hline \multirow{2}{*}{$\begin{array}{c}\text { SFC } \\
(\% \\
w / w)\end{array}$} & \multicolumn{6}{|c|}{$\begin{array}{c}\mathrm{k} \\
\left(\mathrm{day}^{-1}\right)\end{array}$} \\
\hline & $\mathrm{MG}$ & $\mathrm{R}_{\text {adj }}^{2}$ & PPP & $\mathrm{R}_{\text {adj }}^{2}$ & SSS & $\mathrm{R}_{\text {adj }}^{2}$ \\
\hline 0 & $-0.284 \pm 0.062^{\mathrm{a}}$ & 0.965 & $-0.284 \pm 0.062^{b}$ & 0.965 & $-0.284 \pm 0.062^{b}$ & 0.965 \\
\hline 10 & $-0.002 \pm 0.003^{\mathrm{c}, \mathrm{B}}$ & 0.615 & $-0.337 \pm 0.004^{\mathrm{b}, \mathrm{A}}$ & 0.998 & $-0.316 \pm 0.027^{\mathrm{b}, \mathrm{A}}$ & 0.971 \\
\hline 30 & $-0.007 \pm 0.000^{\mathrm{c}, \mathrm{B}}$ & 0.998 & $-0.371 \pm 0.035^{\mathrm{b}, \mathrm{A}}$ & 0.974 & $-0.288 \pm 0.031^{\mathrm{b}, \mathrm{A}}$ & 0.967 \\
\hline 70 & $-0.018 \pm 0.005^{\mathrm{b}, \mathrm{B}}$ & 0.757 & $-0.405 \pm 0.067^{\mathrm{ab}, \mathrm{A}}$ & 0.914 & $-0.268 \pm 0.029^{\mathrm{b}, \mathrm{A}}$ & 0.945 \\
\hline 100 & $-0.017 \pm 0.003^{\mathrm{b}, \mathrm{B}}$ & 0.929 & $-0.512 \pm 0.082^{\mathrm{a}, \mathrm{A}}$ & 0.956 & $-0.617 \pm 0.062^{\mathrm{a}, \mathrm{A}}$ & 0.978 \\
\hline
\end{tabular}

$\overline{\mathrm{a}, \mathrm{b}, \mathrm{c}}$ : Means with different letters in the same column are significantly different $(\mathrm{p}<0.05)$.

A,B,C : Means with different letters in the same row are significantly different $(p<0.05)$. 


\section{Figure captions}

Figure 1. Polarized light (PL) micrographs of mixtures containing increasing concentrations of MG, PPP, and SSS in liquid MCT. Scale bar $=100 \mu \mathrm{m}$.

Figure 2. Fractal dimension of mixtures containing MG (•), PPP (घ), and SSS $(\boldsymbol{\Delta})$ in liquid MCT as a function of solid fat content.

Figure 3. Integrated patterns with relevant peak position of the crystallographic reflecting planes belonging to the $00 l$ family of $100 \%$ MG (A), $50 \%$ SSS (B), 10\% PPP (C) containing samples along with their relative sections of the bidimensional patterns.

Figure 4. Normalized $\beta$-carotene residual concentration $\left(C_{t} / C_{0}\right)$ of $100 \% \mathrm{MG}(\bullet)$, PPP (घ), SSS $(\boldsymbol{\Delta})$, and $\mathrm{MCT}(\boldsymbol{\nabla})$ containing samples as a function of time. 


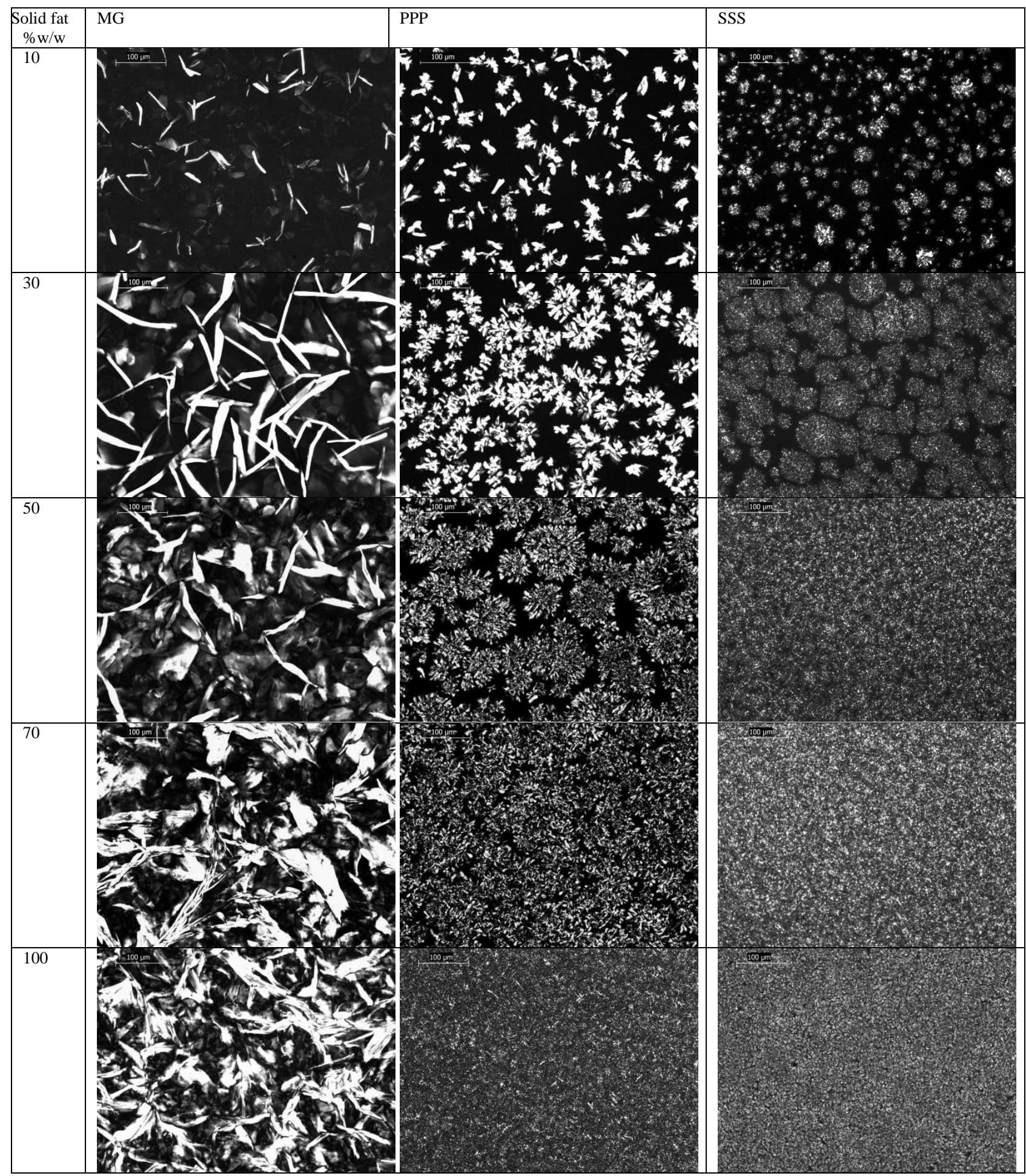




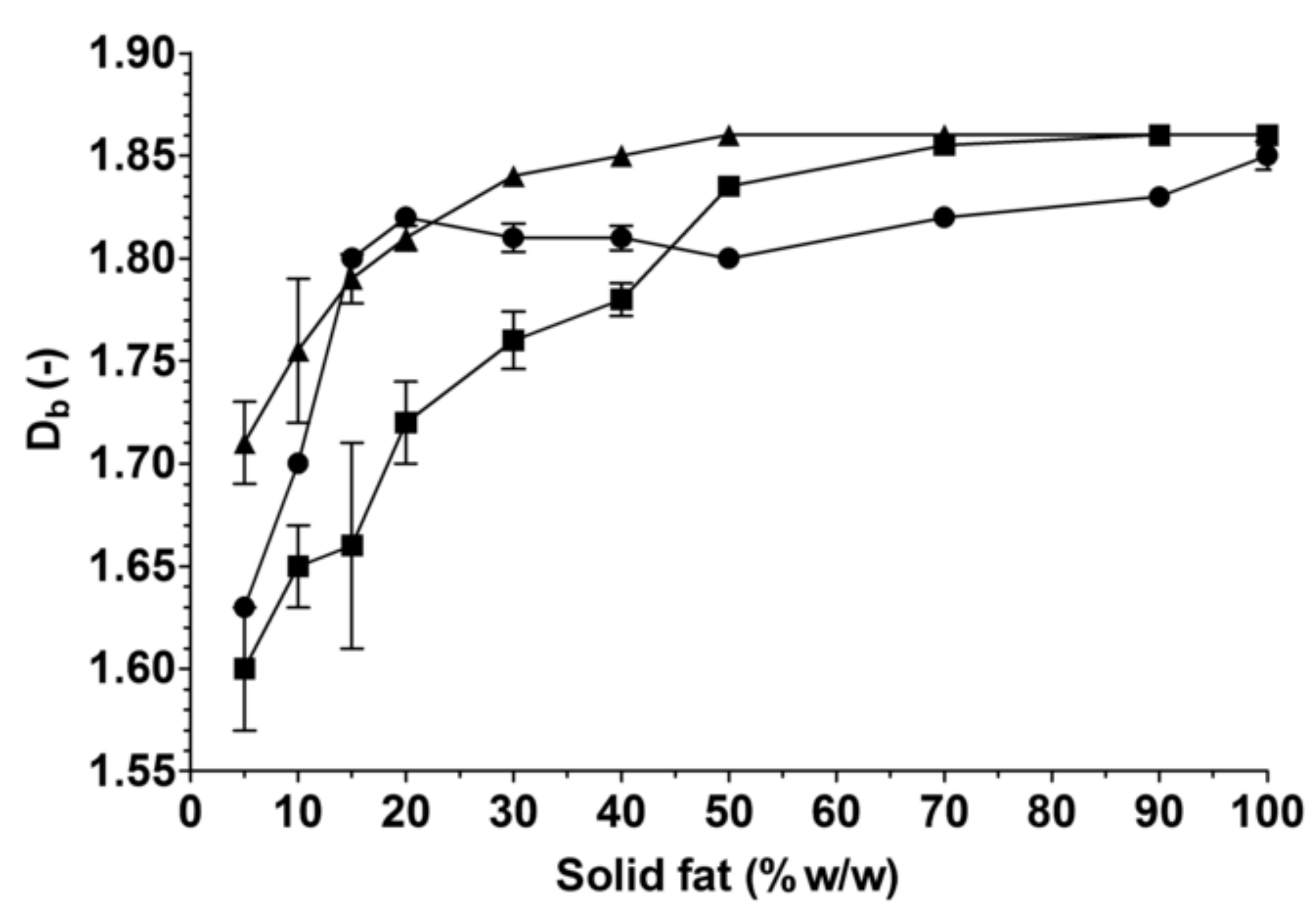

Click here to download high resolution image 

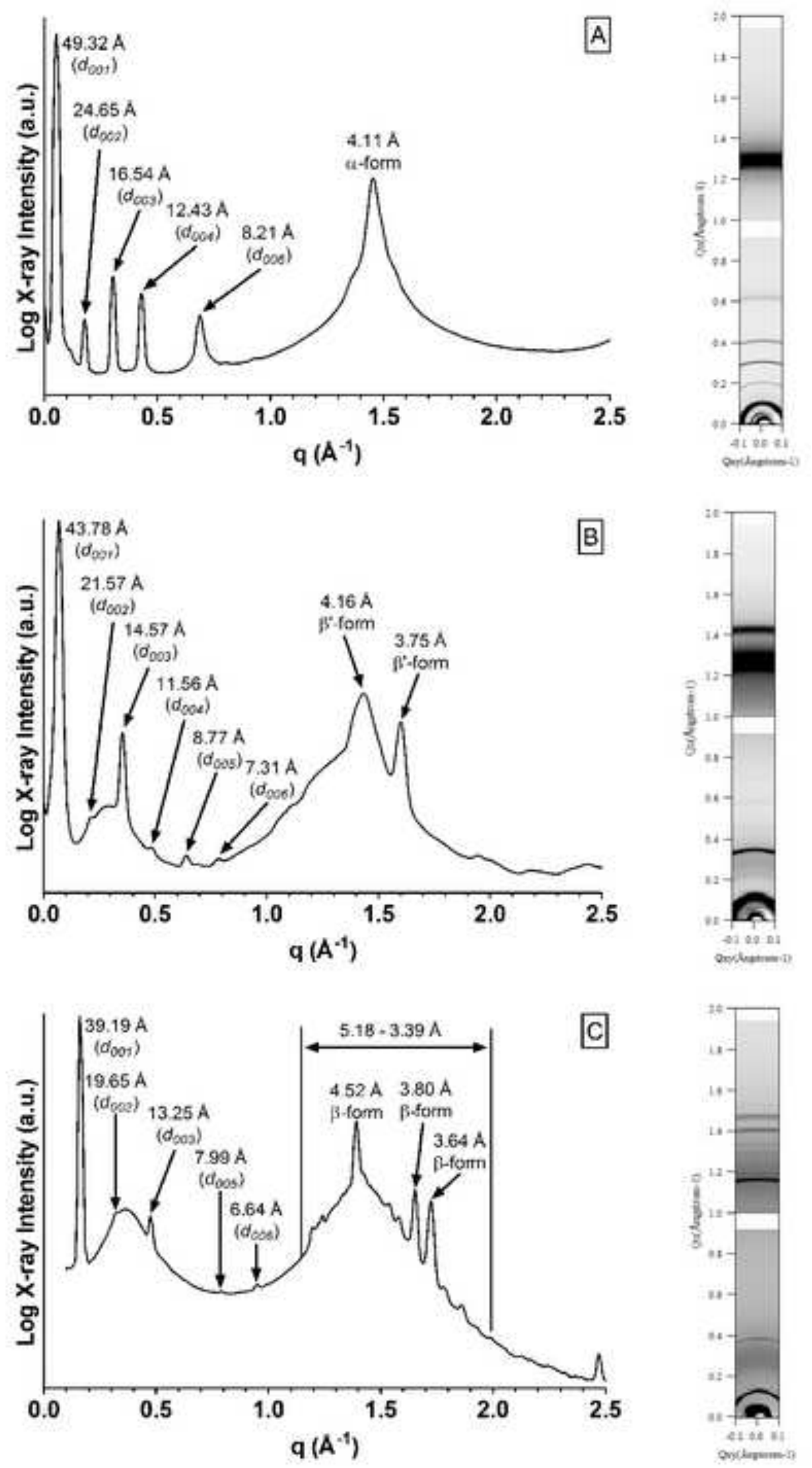


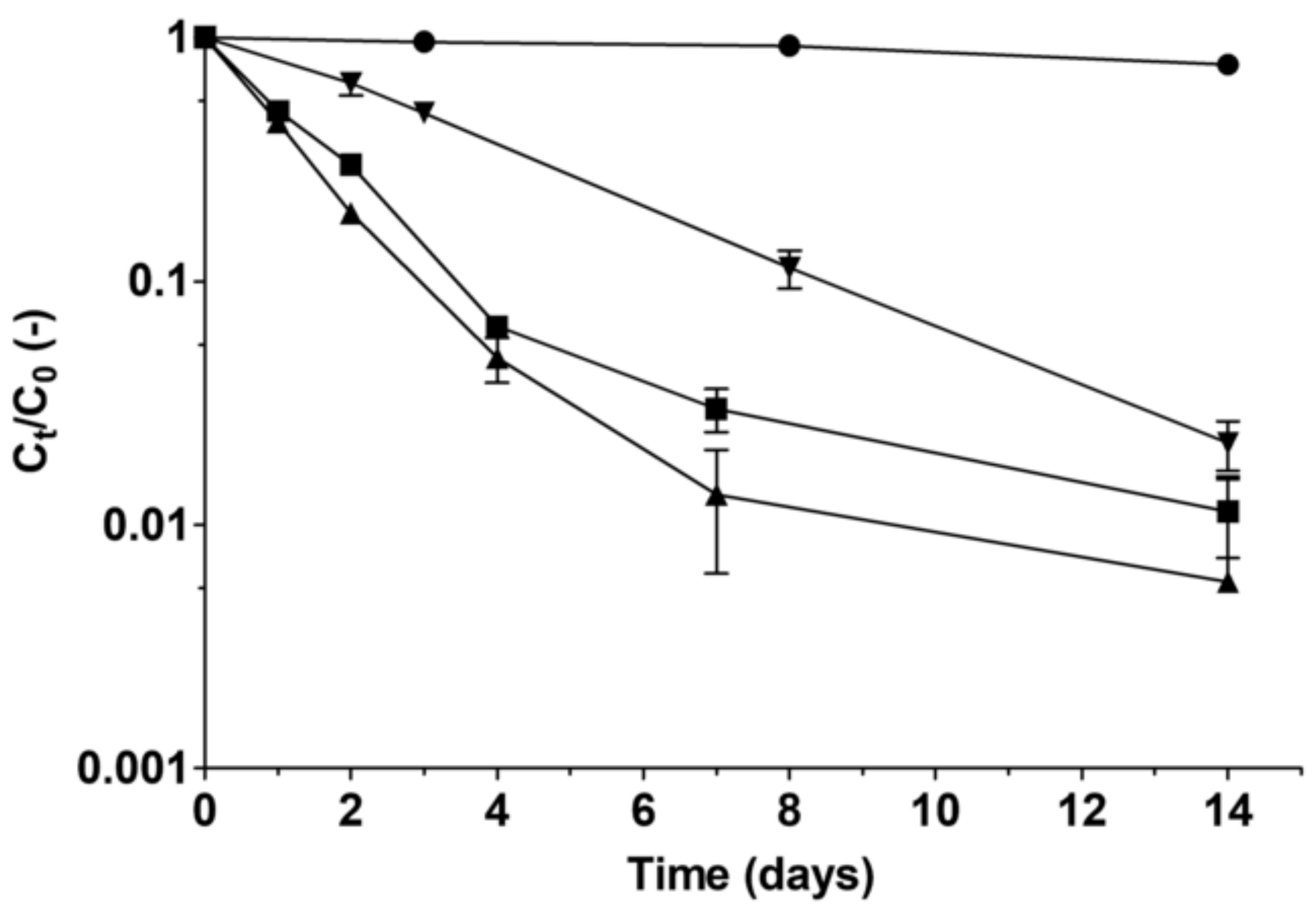




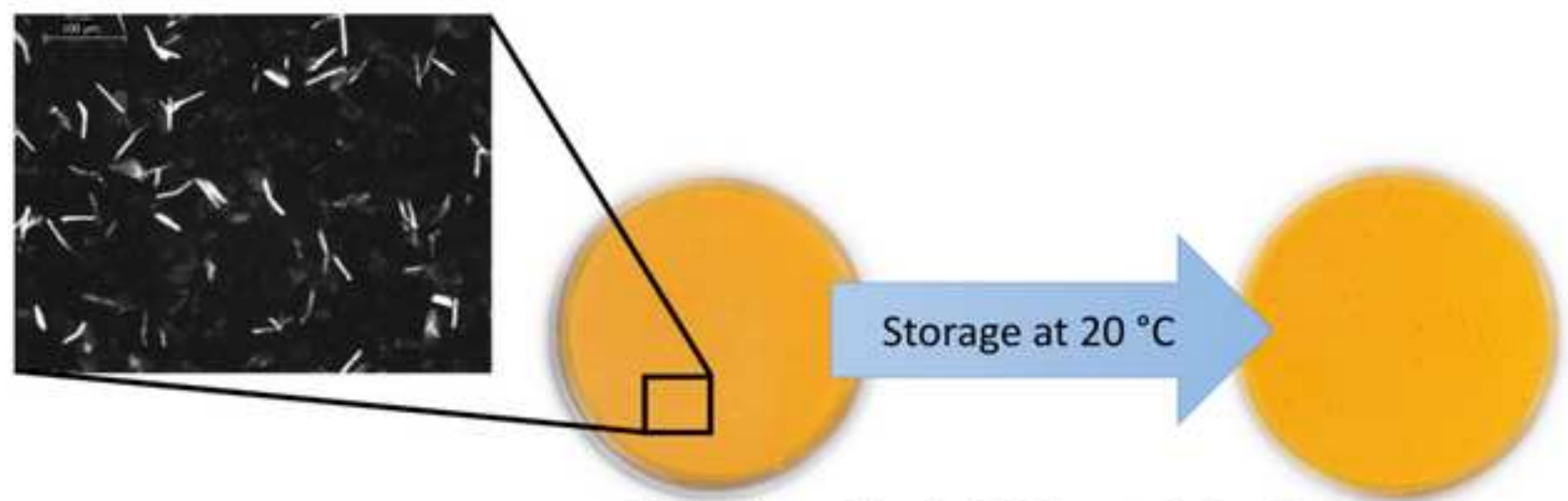

Monoglycerides in MCT containing $\beta$-carotene

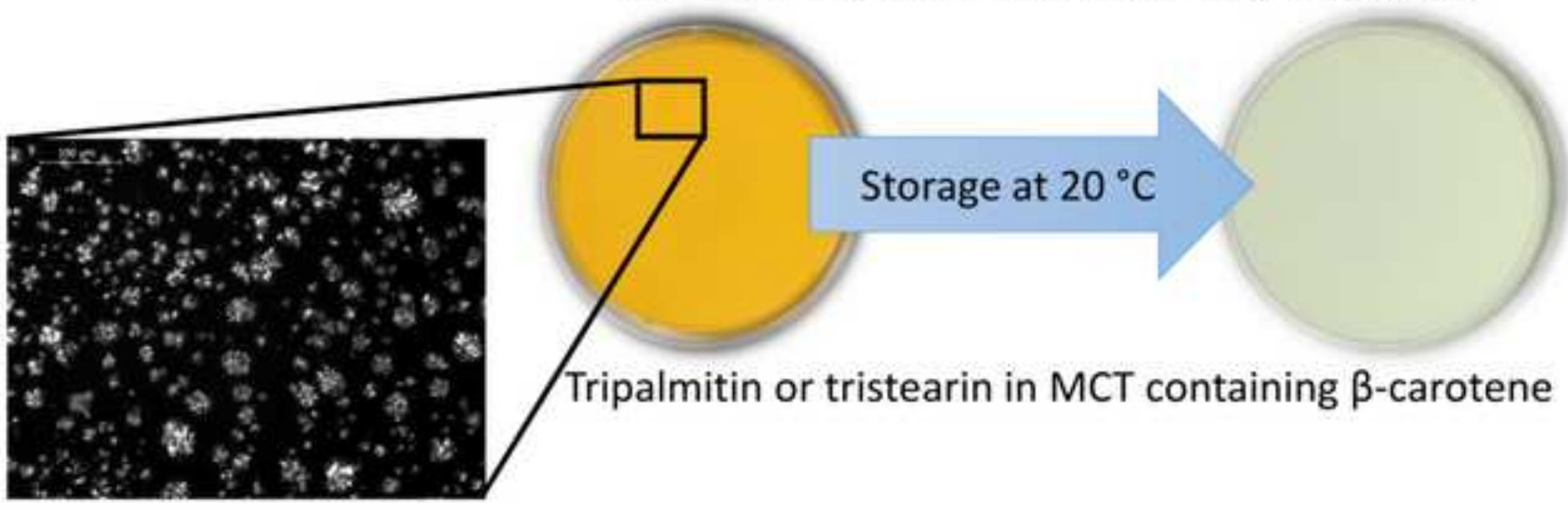

The presence of monoglyceride crystals protects $\beta$-carotene against degradation 